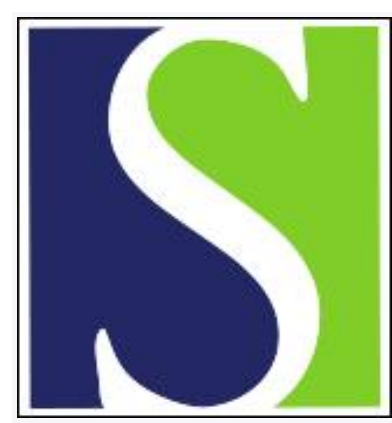

Scand J Work Environ Health 1987;13(6):529-531

https://doi.org/10.5271/sjweh.2004

Issue date: Dec 1987

A remark on the article on tannery workers by Stern et al. by Merler E, Ricci P

Refers to the following text of the Journal: 1987;13(2):108-117

This article in PubMed: www.ncbi.nlm.nih.gov/pubmed/3433055

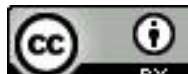


Scand J Work Environ Health 13 (1987) 529-531

\section{A remark on the article on tannery workers by Stern et al}

Stern et al, in a study (4) of a cohort of 9365 tannery workers obtained from the records of two companies, concluded "in general, that leather tanners and finishers are not at an increased risk for those causes of death that were of a priori concern... [p 113]."

Unfortunately, a review of the article suggests that insufficient effort was made to avoid and exclude biases that could have caused negative results. This conclusion is evident, first of all, from the analysis by cumulative years of employment. The authors evaluated the mortality experience for some cancers and restricted the analysis to workers with at least a 15 -year latency interval since first employment. The results revealed a "therapeutic" effect of the tannery environment on cancer mortality. In other words, the standardized mortality ratios decreased as the duration of exposure increased. In the groups with longer exposure, it also decreased steadily for all the cancer sites considered, reaching also the statistical significance of a protective effect for some cancer sites.

The results closely resemble those of Duck et al (1) and Mancuso \& El-Altar (3) in cohort analyses on vinyl chloride and asbestos workers, respectively, which are quoted examples of an inappropriate allocation of person-years $(2,5)$. In a comparison among subgroups, the person-years denominator of the rates must include only the person-years of the same exposure category, and subjects who move through different exposure categories must contribute to the person-years denominators of the different subgroups. When the exposure and follow-up periods overlap, an exact length of observation time must be defined for each subject. It would be better - and even more relevant in the case of negative studies - if computational aspects could also be evaluated by the readers.

If this problem was not pertinent (as it should have been, since a very tested cohort program had been used), the authors should have excluded other possibilities, such as a possible selection bias in the source used to enumerate the study subjects, so that the high percentage of subjects traced would not be relative only to a segment of the workers during the study period. The assumption underlying the choice of the latency period could be inadequate to the specific study question. Only at the end of a similar process can the readers begin to attempt a biological interpretation of such results.

The second methodological aspect is the decision to use only an external standard for the comparison group. In addition to the common problems, the study suggests the presence of other relevant selection phenomena. The authors wrote, for example, that "No information on country of birth (emigration) was available from the employment records [p 110]" and thus pointed out indirectly that the study population was (heavily?) composed of immigrants. The use of local rates does not per se guarantee that the reference group is more similar to that under study, with the exception of the occupational exposures.

The authors also stated that " ... the two tanneries under study had strict enforcement of antismoking rules... [p 115]." No effort was carried out to evaluate the smoking habit of the workers and to estimate the presence of an imbalance of this risk factor between the two populations. A different study design could have avoided the possibiiity of comparing apples with oranges (or apples with pears, as we used to say).

\section{References}

1. Duck BW, Carter JT, Coombes EJ. Mortality study of workers in a polyvinyl-chloride production plant. Lancet 1 (1975) 1197-1199.

2. Enterline PE. Pitfalls in epidemiological research: An examination of the asbestos literature. J Occup Med 18 (1976) $150-156$.

3. Mancuso TF, El-Attar AA. Mortality pattern in a cohort of asbestos workers. J Occup Med 9 (1967) 147-162.

4. Stern FB, Beaumont JJ, Halperin WE, Murthy LI, Hills BW, Fajen JM. Mortality of chrome leather tannery workers and chemical exposures in tanneries. Scand $\mathbf{J}$ Work Environ Health 13 (1987) 108-117.

5. Wagoner JK, Infante PF, Saracci R. Vinyl chloride and mortality? Lancet 1 (1976) 194-195.

Enzo Merler, MD, Paolo Ricci, MD

Institute of Pathology

University of Verona

Centro Ospedaliero Clinicizzato "Borgo Roma"

37134 Verona Italy

\section{Author's reply}

I have read the comments by Drs Enzo Merler and Paolo Ricci regarding the article which I co-authored in the Scandinavian Journal of Work Environment and Health [13 (1987) 108-117] entitled, "Mortality of Chrome Leather Tannery Workers and Chemical Exposures in Tanneries." My response to these comments follows. 
Comment 1. "The authors ... restricted the analysis to workers with at least a 15 -year latency interval since first employment. The results revealed a 'therapeutic effect' of the tannery environment on cancer mortality."

The paper evaluates the risks of all cancers for all workers for all time periods (see tables 4 and 5 in the original article). The results do not show a "therapeutic effect" of the tannery environment on cancer mortality.

The goal of occupational epidemiology is to delineate the association between occupation and disease. The decision of how to examine the data is dependent on the hypothesis to be tested. In the tannery workers study, it was hypothesized that exposure to the various known or suspected occupational carcinogens among the workers in the tannery environment (hexavalent chromium salts, benzidine-based azo dyes, aromatic organic solvents, formaldehyde, airborne leather dust, and nitrosamines) could be associated with an increased risk of mortality due to various cancers (lung, larynx, bladder, kidney, nasal cavity, etc) as reported by others for these exposures. A statement on this recognized association was made in the first sentence of the paper. It is standard procedure in a cohort study to examine the influence of increased exposure and latency. The risk for various cancers should increase with length of exposure (employment), and the risk should become evident only after a substantial time period between exposure and disease (latency). It has been generally accepted that cohort mortality studies aimed at estimating relative risk for these cancers should allow for 15 years of latency or longer (reference number 8 in the article). This general practice is pointed out on page 110 , second column of the article. No increase in cancer risk with increasing duration of employment after 15 years' latency was found, as shown in table 5 of the article. However, in the study, we examined workers with increasing duration of exposure within latency periods of less than 15 years and also found no increase in cancer mortality risk.

Comment 2. ". . . inappropriate allocation of personyears. In a comparison among subgroups, the personyears denominator of the rates must include only the person-years of the same exposure category, and subjects who move through different exposure categories must contribute to the person-years denominators of the different subgroups."

Drs Merler and Ricci misunderstood our life-table methods, which are described in the Waxweiler reference (reference number 41 in the article). As the article points out, the life-table analysis system of the National Institute for Occupational Safety and Health uses the "critical date" concept to allocate person-years to various cells. "Critical dates" are the dates at which a worker moves across a cell (exposure and/or latency). The program can then accumulate various cells of person-years as the analysis warrants. The personyears denominator of the rates only includes personyears of the same exposure and latency categories, and, as subjects move through different exposure and latency categories, they contribute person-years to the denominator of the different exposure and latency subgroups only.

Comment 3. "...the high percentage of subjects traced would not be relative..." and should not have been included in the study.

If Drs Merler and Ricci are stating that complete follow-up is not necessary, we disagree. In a cohort study, all tannery workers, no matter how long employed, should be included in the study, since all tannery workers were potentially exposed. Trends for different variables (exposure and latency) can then be analyzed to determine whether mortality ratios increase with increasing worker exposure and latency.

Comment 4. "... decision to use only an external standard for the comparison group."

In our analysis of tannery workers, we had two potential choices of a comparison group. First, we could have used workers in the cohort who were not exposed. However we were unable to use an internal comparison in our study because there were not enough unexposed workers at the tannery to form a cohort with similar variables of age, race, sex, and calendar-time period. The second choice was to use a generalized population. It was felt that state rates would be better than national rates for the following two reasons: (i) in the United States, causes of death are not equally distributed by state, especially in the dairy farm areas of Minnesota and Wisconsin, (ii) Wisconsin and Minnesota may have different distributions of ethnic groups, as compared to the United States as a whole.

Comment 5. ". . . No information on country of birth (emigration) was available from employment records [p 110]' and thus pointed out indirectly that the study population was (heavily?) composed of immigrants."

We did not know the countries in which members of the study population were born. However, this is the case in most cohort mortality studies. The readers, nonetheless, are not to assume that most of the workers were born outside the United States.

Comment 6. ". . . no effort was carried out to evaluate the smoking habits of the workers and to estimate the 
presence of an imbalance of this risk factor between the two populations."

The effect of cigarette smoking could be a source of uncontrolled confounding if there has been an unequal distribution of smokers between the cohort and the comparison population. On one hand, we know that the two tanneries had strict enforcement of antismoking rules, and, therefore, employees may not have smoked as much as the comparison population. On the other, we are aware of various studies which suggest that blue-collar workers actually smoke more than the United States population as a whole, which would have had the effect of artificially increasing the already low standardized mortality ratios. However, as stated in the original article, smoking histories for the cohort were not available, and, therefore, analyses for the confounding of smoking could not be carried out.

Frank B Stern, MS

Epidemiology Section I

Industrywide Studies Branch

Division of Surveillance, Hazard Evaluations and Field Studies

National Institute for Occupational Safety and Health

Robert A Taft Laboratories

4676 Columbia Parkway

Cincinnati OH 45226 USA 